\title{
Highly diastereoselective Diels- Alder reaction using a chiral auxiliary derived from levoglucosenone
}

\author{
Ariel M. Sarotti, Rolando A. Spanevello and Alejandra G. Suárez* \\ Instituto de Química Orgánica de Síntesis, Facultad de Ciencias Bioquímicas y \\ Farmacéuticas, Universidad Nacional de Rosario - CONICET Suipacha 531, \\ S2002LRK Rosario, Argentina \\ asuarez@ fbioyf.unr.edu.ar
}
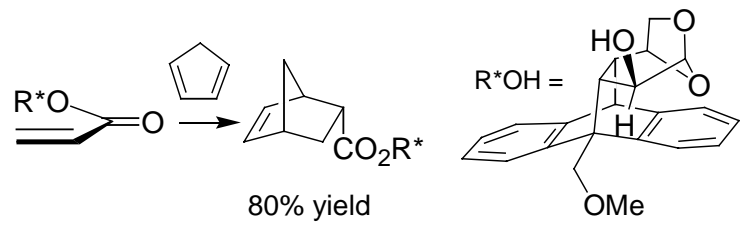

$98 \%$ de 


\section{General}

The melting points were taken on a Leitz Wetzlar Microscope Heating Stage Model 350 apparatus and are uncorrected. Optical rotations were recorded with a Jasco DIP 1000 polarimeter. Infrared spectra were obtained on an IRPrestige-21 Fourier Transform Spectrophotometer Shimadsu. High resolution mass spectrometry measurements were performed using a Waters AutoSpect equipment or Applied Biosystems MS. HPLC analyses were performed with a chromatograph Varian ProStar equipped with UV-V detector ProStar 320 at $270 \mathrm{~nm}$. HPLC was performed on a Beckman C-18, $25 \mathrm{~cm}$ column. Acetonitrile and water HPLC grade was used as eluent in a mixture 60:40 respectively. Flow rate was $2 \mathrm{~mL} / \mathrm{min}$. Nuclear magnetic resonance spectra were recorded on a Bruker AC-200 spectrometer with tetramethylsilane as internal standard and deuterochloroform as solvent. The NMR assignments were corroborated by NOE measurements, $\mathrm{H}, \mathrm{H}$ - and $\mathrm{H}, \mathrm{C}$-correlations.

The reactions were monitored by thin layer chromatography carried out on $0.25 \mathrm{~mm}$ E. Merck silica gel plates (60F254) that were developed using UV light and anisaldehydesulfuric acid-acetic acid with subsequent heating. Flash column chromatography were performed using Merck silica gel $60 \mathrm{H}$, by gradient elution created by mixtures of hexanes and increasing amounts of ethyl acetate.

All reactions were carried out under argon atmosphere with dry, freshly distilled solvents under anhydrous conditions unless otherwise noted. Yields refer to chromatographically and spectroscopically $\left({ }^{1} \mathrm{H}\right.$ NMR) homogeneous materials, unless otherwise stated.

\section{Preparation of compound 1}

Levoglucosenone was synthesized according to the procedure described in literature. ${ }^{1}$

\section{Preparation of compound 3}

This compound was prepared by a modification of the published procedure. ${ }^{2}$ 9Anthracenemethanol 97\% (6.09 g, $28.38 \mathrm{mmol})$ was dried azeotropically with dry benzene and dissolved in THF $(96 \mathrm{~mL})$ under argon. This solution was cooled at $0^{\circ} \mathrm{C}$ and $\mathrm{NaH}(60 \%, 3.51 \mathrm{~g}, 87.83 \mathrm{mmol})$ was added and stirred for 20 minutes. Methyl iodide $(4.4 \mathrm{~mL}, 70.71 \mathrm{mmol})$ and tetrabutyl ammonio iodide $(1.04 \mathrm{~g}, 2.82 \mathrm{mmol})$ were added, the final solution was stirred for 40 minutes and allowed to reach room temperature. This solution was cooled at $0^{\circ} \mathrm{C}$ and carefully added methanol $(5 \mathrm{~mL})$ and diluted with AcOEt $(1 \mathrm{~L})$. The organic phase was washed with water $(2 \times 100 \mathrm{~mL})$, brine $(100 \mathrm{~mL})$, dried $\left(\mathrm{Na}_{2} \mathrm{SO}_{4}\right)$ and concentrated. The residual solid was purified by flash chromatography to afford 3 (6.06 g, $27.25 \mathrm{mmol}, 96 \%)$.

\footnotetext{
${ }^{1}$ Witczak, Z. J. (Ed.), Levoglucosenone and Levoglucosans: Chemistry and Applications, ATL Press, Mount Prospect, 1994. Chapter 2. (Morin, C.)

${ }^{2}$ Zimmermman, C.; Mohr, M.; Zipse, H.; Eichberger, R.; Schnabel, W. J. Photochem. Photobiol. A. 1999, 125, 47.
} 


\section{Compound 3:}

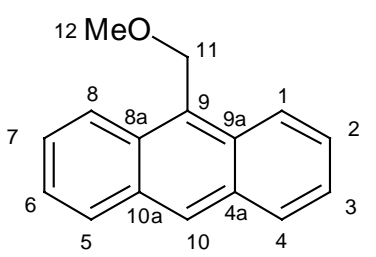

3

Yellow cristalline solid; $\mathrm{mp}=91-92{ }^{\circ} \mathrm{C}$ (hexane/ethyl acetate); IR $(\mathrm{KBr}) v_{\text {máx }}: 2977$, 2924, 1623, 1447, 1344, 1188, 1087, 940, 894, 738, $729 \mathrm{~cm}^{-1} ;{ }^{1} \mathrm{H}$ NMR $\left(\mathrm{CDCl}_{3}\right) \delta 8.42$ (s, $1 \mathrm{H}, \mathrm{H}-10), 8.36$ (d, J=8.8 Hz, $2 \mathrm{H}, \mathrm{H}-1$ and H-8), 7.98 (d, J=8.4 Hz, $2 \mathrm{H}, \mathrm{H}-4$ and H-5), 7.56 - 7.40 (m, 4 H, H-2, H-3, H-6 and H-7), 5.40 (s, 2 H, H-11), 3.51 (s, 3 H, H12); ${ }^{13} \mathrm{C}$ NMR $\left(\mathrm{CDCl}_{3}\right) \delta 131.3(\mathrm{C}, \mathrm{C}-9), 130.8(\mathrm{C}, \mathrm{C}-4 \mathrm{a}$ and $\mathrm{C}-10 \mathrm{a}), 128.8(\mathrm{CH}, \mathrm{C}-4$ and C-5), 128.5 (C, C-8a and C-9a), 128.2 (CH, C-10), 126.0 (CH, C-1 and C-8), 124.8 $(\mathrm{CH}, \mathrm{C}-2$ and $\mathrm{C}-7), 124.1\left(\mathrm{CH}, \mathrm{C}-3\right.$ and C-6), $66.4\left(\mathrm{CH}_{2}, \mathrm{C}-11\right), 58.1\left(\mathrm{CH}_{3}, \mathrm{C}-12\right)$.

\section{Cycloaddition reaction of 1 and 3 .}

Levoglucosenone 96\% (1.54 g, $11.74 \mathrm{mmol})$ and 9-methoxymethylanthracene (7.19 g, $32.36 \mathrm{mmol})$ were dissolved in toluene $(51 \mathrm{~mL})$ at room temperature. The solution was heated under reflux during seven days and evaporated under vacuum. The solid residue was purified by flash chromatography to give $4(3.54 \mathrm{mg}, 10.16 \mathrm{mmol}, 87 \%)$ and 5 $(0.47 \mathrm{~g}, 1.36 \mathrm{mmol}, 12 \%)$. The excess of the diene was recovered quantitatively and reused.

\section{Cycloadduct 4}

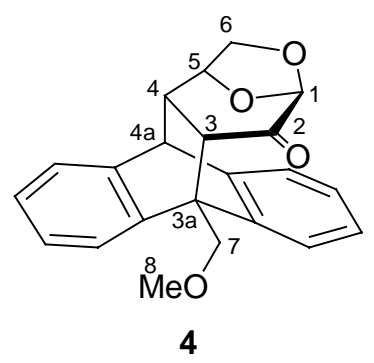

White cristalline solid; $\mathrm{mp}=251-252{ }^{\circ} \mathrm{C}$ (hexane/chloroform); $[\alpha]_{\mathrm{D}}=-43.8(\mathrm{c}=0.88$; $\mathrm{CHCl}_{3}$ ); IR (KBr) v $v_{\text {máx }}:$ 3069, 2927, 1719 (C=O), 1464, 1457, 1115, 993, 921, 770 $\mathrm{cm}^{-1} ;{ }^{1} \mathrm{H} \mathrm{NMR}\left(\mathrm{CDCl}_{3}\right) \delta$ 7.51-7.00 (m, $8 \mathrm{H}$, aromatics), $4.89\left(\mathrm{~d}, J_{\text {gem }}=9.5 \mathrm{~Hz}, 1 \mathrm{H}, \mathrm{H}-\right.$ 7), $4.77\left(\mathrm{~d}, J_{5-6 \mathrm{exo}}=4.9 \mathrm{~Hz}, 1 \mathrm{H}, \mathrm{H}-5\right), 4.62$ (d, $\left.J_{\text {gem }}=9.5 \mathrm{~Hz}, 1 \mathrm{H}, \mathrm{H}-7\right), 4.54$ (s, $\left.1 \mathrm{H}, \mathrm{H}-1\right)$, $4.27(\mathrm{~s}, 1 \mathrm{H}, \mathrm{H}-4 \mathrm{a}), 3.76-3.61(\mathrm{~m}, 5 \mathrm{H}, \mathrm{H}-6$ endo, H-6 exo and H-8), 3.10 (d, J3-4=9.7 $\mathrm{Hz}, 1 \mathrm{H}, \mathrm{H}-3), 2.24$ (d, $\left.J_{3-4}=9.7 \mathrm{~Hz}, 1 \mathrm{H}, \mathrm{H}-4\right) ;{ }^{13} \mathrm{C} \mathrm{NMR}\left(\mathrm{CDCl}_{3}\right) \delta 199.1(\mathrm{C}, \mathrm{C}-2)$, 145.2 (C, aromatic.), 141.3 (C, aromatic), 140.9 (C, aromatic), 140.6 (C, aromatic), $126.2(\mathrm{CH}$, aromatic), $126.0(\mathrm{CH}, 2 \mathrm{C}$, aromatic $), 125.8(\mathrm{CH}$, aromatic $), 125.0(\mathrm{CH}$, aromatic), $124.2(\mathrm{CH}$, aromatic), $122.0(\mathrm{CH}$, aromatic), $121.3(\mathrm{CH}$, aromatic $), 99.8$ (CH, C-1), $77.1(\mathrm{CH}, \mathrm{C}-5), 70.5\left(\mathrm{CH}_{2}, \mathrm{C}-7\right), 68.7\left(\mathrm{CH}_{2}, \mathrm{C}-6\right), 59.0\left(\mathrm{CH}_{3}, \mathrm{C}-8\right), 50.3$ $(\mathrm{CH}, \mathrm{C}-4 \mathrm{a}), 49.8$ (C, C-3a), 44.7 (CH, C-4), $44.0(\mathrm{CH}, \mathrm{C}-3)$. 


\section{Cycloadduct 5}

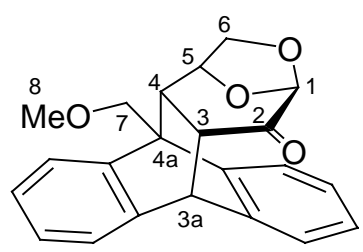

5

White cristalline solid; $\mathrm{mp}=236-237^{\circ} \mathrm{C}$ (hexane/chloroform); $[\alpha]_{\mathrm{D}}=-127.7(\mathrm{c}=0.92$; $\left.\mathrm{CHCl}_{3}\right)$; IR (KBr) v váx $: 3062,2969,1735(\mathrm{C}=\mathrm{O}), 1457,1118,1108,983,746 \mathrm{~cm}^{-1} ;{ }^{1} \mathrm{H}$ NMR $\left(\mathrm{CDCl}_{3}\right) \delta$ 7.36-7.03 (m, $8 \mathrm{H}$, aromatics), $4.88\left(\mathrm{~d}, J_{5-6 \text { exo }}=5.4 \mathrm{~Hz}, 1 \mathrm{H}, \mathrm{H}-5\right), 4.84$ $\left(\mathrm{d}, J_{3-3 \mathrm{a}}=3.4 \mathrm{~Hz}, 1 \mathrm{H}, \mathrm{H}-3 \mathrm{a}\right), 4.69$ (d, $\left.J_{\mathrm{gem}}=9.9 \mathrm{~Hz}, 1 \mathrm{H}, \mathrm{H}-7\right), 4.64$ (s, $\left.1 \mathrm{H}, \mathrm{H}-1\right), 4.62$ (d, $\left.J_{\text {gem }}=9.9 \mathrm{~Hz}, 1 \mathrm{H}, \mathrm{H}-7\right), 3.84\left(\mathrm{dd}, J_{\text {gem }}=7.2 \mathrm{~Hz}, J_{5-6 \text { exo }}=5.4 \mathrm{~Hz}, 1 \mathrm{H}, \mathrm{H}-6\right.$ exo $), 3.72-3.68$ (m, $4 \mathrm{H}, \mathrm{H}-6$ endo and H-8), $2.99\left(\mathrm{dd}, J_{3-4}=9.9 \mathrm{~Hz}, J_{3-3 \mathrm{a}}=3.4 \mathrm{~Hz}, 1 \mathrm{H}, \mathrm{H}-3\right), 2.42$ (d, $J_{3-}$ $\left.{ }_{4}=9.9 \mathrm{~Hz}, 1 \mathrm{H}, \mathrm{H}-4\right) ;{ }^{13} \mathrm{C} \mathrm{NMR}\left(\mathrm{CDCl}_{3}\right) \delta 199.0(\mathrm{C}, \mathrm{C}-2), 144.5(\mathrm{C}$, aromatic), $141.5(\mathrm{C}$, aromatic), $141.2(\mathrm{C}$, aromatic), $140.3(\mathrm{C}$, aromatic), $126.2(\mathrm{CH}$, aromatic), $125.9(\mathrm{CH}$, $3 \mathrm{C}$, aromatic), $124.9(\mathrm{CH}$, aromatic $), 124.0(\mathrm{CH}$, aromatic $), 121.8(\mathrm{CH}$, aromatic $), 121.4$ $\left(\mathrm{CH}\right.$, aromatic), $99.4(\mathrm{CH}, \mathrm{C}-1), 72.7(\mathrm{CH}, \mathrm{C}-5), 70.4\left(\mathrm{CH}_{2}, \mathrm{C}-7\right), 69.4\left(\mathrm{CH}_{2}, \mathrm{C}-6\right)$, $59.5\left(\mathrm{CH}_{3}, \mathrm{C}-8\right), 47.7(\mathrm{C}, \mathrm{C}-4 \mathrm{a}), 46.3(\mathrm{CH}, \mathrm{C}-3 \mathrm{a}), 45.4(\mathrm{CH}, \mathrm{C}-4), 43.7(\mathrm{CH}, \mathrm{C}-3)$.

\section{Reduction of cycloadduct 4}

Cycloadduct $4(2755 \mathrm{mg}, 7.91 \mathrm{mmol})$ was dissolved in a $\mathrm{CH}_{2} \mathrm{Cl}_{2}$ : $\mathrm{MeOH}$ 95:5 mixture $(154 \mathrm{~mL})$, cooled at $0^{\circ} \mathrm{C}$ and $\mathrm{NaBH}_{4}(606 \mathrm{mg}, 16.02 \mathrm{mmol})$ was incorporated. The mixture was stirred during $5 \mathrm{~h}$ and then water $(50 \mathrm{~mL})$ was added. The solution was extracted with $\mathrm{CH}_{2} \mathrm{Cl}_{2}(100 \mathrm{~mL})$ and then with AcOEt $(3 \times 100 \mathrm{~mL})$. The combined organic extracts were dried $\left(\mathrm{Na}_{2} \mathrm{SO}_{4}\right)$ and concentrated. The residue was purified by flash chromatography to afford 6 (1744 $\mathrm{mg}, 4.98 \mathrm{mmol}, 63 \%)$ and 7 (737 $\mathrm{mg}, 2.10$ mmol, 27\%).

Alcohol 6

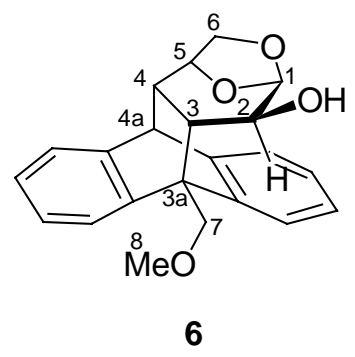

White solid; $\mathrm{mp}=196-197{ }^{\circ} \mathrm{C}$ (hexane/dichloromethane); $[\alpha]_{\mathrm{D}}=+19.7(\mathrm{c}=1.11$; $\mathrm{CHCl}_{3}$ ); IR (KBr) $v_{\text {máx }}: 3406,3072,2888,1456,1101,992,944,748 \mathrm{~cm}^{-1} ;{ }^{1} \mathrm{H}$ NMR $\left(\mathrm{CDCl}_{3}\right) \delta$ 7.43-7.03 (m, $8 \mathrm{H}$, aromatics), $4.98\left(\mathrm{~d}, J_{1-2}=3.2 \mathrm{~Hz}, 1 \mathrm{H}, \mathrm{H}-1\right), 4.63(\mathrm{~d}$, $\left.J_{5-6 \mathrm{exo}}=4.9 \mathrm{~Hz}, 1 \mathrm{H}, \mathrm{H}-5\right), 4.57\left(\mathrm{~d}, J_{\mathrm{gem}}=9.9 \mathrm{~Hz}, 1 \mathrm{H}, \mathrm{H}-7\right), 4.45\left(\mathrm{~d}, J_{\mathrm{gem}}=9.9 \mathrm{~Hz}, 1 \mathrm{H}, \mathrm{H}-\right.$ 7), 4,13 (s, $1 \mathrm{H}, \mathrm{H}-4 \mathrm{a}), 3.71$ (dd, $J_{\text {gem }}=7.1 \mathrm{~Hz}, J_{5-6 \text { exo }}=4.9 \mathrm{~Hz}, 1 \mathrm{H}, \mathrm{H}-6$ exo), 3.66 (s, 3 
H, H-8), 3.60 (d, $J_{\text {gem }}=7.1 \mathrm{~Hz}, 1 \mathrm{H}, \mathrm{H}-6$ endo), $2.93\left(\mathrm{dd}, J_{2-3}=5.8 \mathrm{~Hz}, J_{1-2}=3.2 \mathrm{~Hz}, 1 \mathrm{H}\right.$, H-2), 2.44 (broad s, $1 \mathrm{H}, \mathrm{OH}), 2.20$ (dd, $\left.J_{3-4}=10.5 \mathrm{~Hz}, J_{2-3}=5.8 \mathrm{~Hz}, 1 \mathrm{H}, \mathrm{H}-3\right), 2.04$ (d, $\left.J_{3-4}=10.5 \mathrm{~Hz}, 1 \mathrm{H}, \mathrm{H}-4\right) ;{ }^{13} \mathrm{C} \mathrm{NMR}\left(\mathrm{CDCl}_{3}\right) \delta 146.3$ (C, aromatic), 141.5 (C, aromatic), 141.1 (C, aromatic), 140.8 (C, aromatic), 126.1 ( $\mathrm{CH}$, aromatic), 125.7 ( $\mathrm{CH}$, aromatic), $125.6(\mathrm{CH}$, aromatic), $125.5(\mathrm{CH}$, aromatic $), 124.9(\mathrm{CH}$, aromatic $), 123.5(\mathrm{CH}$, aromatic), $122.9(\mathrm{CH}$, aromatic), $121.8(\mathrm{CH}$, aromatic), $99.8(\mathrm{CH}, \mathrm{C}-1), 76.6(\mathrm{CH}, \mathrm{C}-5)$, $71.6\left(\mathrm{CH}_{2}, \mathrm{C}-7\right), 70.3\left(\mathrm{CH}_{2}, \mathrm{C}-6\right), 68.7(\mathrm{CH}, \mathrm{C}-2), 59.0\left(\mathrm{CH}_{3}, \mathrm{C}-8\right), 50.3(\mathrm{CH}, \mathrm{C}-4 \mathrm{a})$, 49.9 (C, C-3a), $47.6(\mathrm{CH}, \mathrm{C}-4), 41.6(\mathrm{CH}, \mathrm{C}-3)$; HRMS calc. for $\mathrm{C}_{22} \mathrm{H}_{23} \mathrm{O}_{4}[\mathrm{M}+\mathrm{H}]^{+}$ 351.1596 , found 351.1581 .

\section{Alcohol 7}

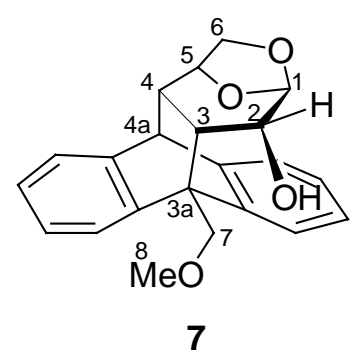

White cristalline solid; $\mathrm{mp}=207-208{ }^{\circ} \mathrm{C}$ (hexane/dichloromethane); $[\alpha]_{\mathrm{D}}=+103.2$ (c=1.24; $\left.\mathrm{CHCl}_{3}\right)$; IR (KBr) $v_{\text {máx }}: 3565,2941,2887,1464,1115,1038,998,756,743$ $\mathrm{cm}^{-1} ;{ }^{1} \mathrm{H} \mathrm{NMR}\left(\mathrm{CDCl}_{3}\right) \delta$ 7.43-6.99 (m, $8 \mathrm{H}$, aromatics), $4.99(\mathrm{~s}, 1 \mathrm{H}, \mathrm{H}-1), 4.84(\mathrm{~d}$, $\left.J_{\text {gem }}=9.2 \mathrm{~Hz}, 1 \mathrm{H}, \mathrm{H}-7\right), 4.68$ (d, $\left.J_{\text {gem }}=9.2 \mathrm{~Hz}, 1 \mathrm{H}, \mathrm{H}-7\right), 4.62(\mathrm{~s}, 1 \mathrm{H}, \mathrm{H}-5), 4.10(\mathrm{~s}, 1 \mathrm{H}$, H-4a), 3.88 (dd, $\left.J_{2-\mathrm{OH}}=12.3 \mathrm{~Hz}, J_{2-3}=7.9 \mathrm{~Hz}, 1 \mathrm{H}, \mathrm{H}-2\right), 3.66-3.64$ (m, $5 \mathrm{H}, \mathrm{H}-6$ exo, H-6 endo and H-8), 2.69 (dd, $\left.J_{3-4}=10.9 \mathrm{~Hz}, J_{2-3}=7.9 \mathrm{~Hz}, 1 \mathrm{H}, \mathrm{H}-3\right), 1.94\left(\mathrm{~d}, J_{3-4}=10.9 \mathrm{~Hz}, 1\right.$ $\mathrm{H}, \mathrm{H}-4), 0.77\left(\mathrm{~d}, J_{2-\mathrm{OH}}=12.3 \mathrm{~Hz}, 1 \mathrm{H}, \mathrm{OH}\right) ;{ }^{13} \mathrm{C} \mathrm{NMR}\left(\mathrm{CDCl}_{3}\right) \delta 147.4$ (C, aromatic), 144.1 (C, aromatic), 142.4 (C, aromatic), 141.1 (C, aromatic), $125.6(\mathrm{CH}, 3 \mathrm{C}$, aromatic), $125.4(\mathrm{CH}$, aromatic), $125.0(\mathrm{CH}$, aromatic), $123.4(\mathrm{CH}$, aromatic), 122.8 $\left(\mathrm{CH}\right.$, aromatic), $121.6\left(\mathrm{CH}\right.$, aromatic), $102.3(\mathrm{CH}, \mathrm{C}-1), 76.3(\mathrm{CH}, \mathrm{C}-5), 72.0\left(\mathrm{CH}_{2}, \mathrm{C}-\right.$ 7), $71.2\left(\mathrm{CH}_{2}, \mathrm{C}-6\right), 68.3(\mathrm{CH}, \mathrm{C}-2), 59.3\left(\mathrm{CH}_{3}, \mathrm{C}-8\right), 50.8$ (CH, C-4a), 49.7 (C, C-3a), $45.3(\mathrm{CH}, \mathrm{C}-4), 34.3(\mathrm{CH}, \mathrm{C}-3)$.

\section{Preparation of acrylate 8}

Alcohol 6 (797 mg, $2.27 \mathrm{mmol}$ ) was dried azeotropically with dry benzene, dissolved in $\mathrm{CH}_{2} \mathrm{Cl}_{2}(32 \mathrm{~mL})$ and cooled at $0^{\circ} \mathrm{C}$. Triethylamine $(1.09 \mathrm{~mL}, 7.81 \mathrm{mmol})$ and acryloyl chloride $(0.42 \mathrm{~mL}, 5.17 \mathrm{mmol})$ were added and stirred for $1 \mathrm{~h}$ under argon atmosphere. The mixture was diluted with water $(50 \mathrm{~mL})$ and extracted several times with $50 \mathrm{~mL}$ portions of $\mathrm{CH}_{2} \mathrm{Cl}_{2}$. The combined organic extracts were dried $\left(\mathrm{Na}_{2} \mathrm{SO}_{4}\right)$ and concentrated. The residue was purified by flash chromatography to give $\mathbf{8}$ (741 $\mathrm{mg}, 1.83$ mmol, $81 \%)$. 


\section{Acrylate 8}

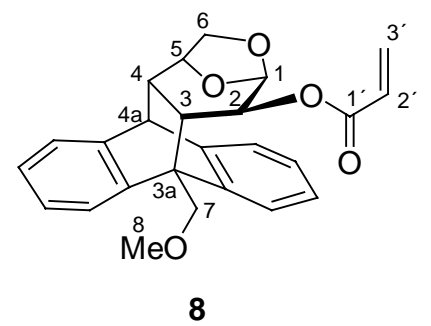

Colorless oil; $[\alpha]_{D}=-12.9\left(\mathrm{c}=1.22 ; \mathrm{CHCl}_{3}\right)$; IR (film) $v_{\text {máx }}: 2893,1719(\mathrm{C}=\mathrm{O}), 1405$, 1196, 1161, 1148, 1113, 1004, $750 \mathrm{~cm}^{-1} ;{ }^{1} \mathrm{H}$ NMR $\left(\mathrm{CDCl}_{3}\right) \delta 7.44-7.04(\mathrm{~m}, 8 \mathrm{H}$, aromatic), $6.52\left(\mathrm{dd}, J_{\mathrm{vic}}=17.2 \mathrm{~Hz}, J_{\mathrm{gem}}=1.6 \mathrm{~Hz}, 1 \mathrm{H}, \mathrm{H}-3^{\prime} c i s\right), 6.21$ (dd, $J_{\text {vic }}=17.2 \mathrm{~Hz}$, $\left.J_{\mathrm{vic}}=10.3 \mathrm{~Hz}, 1 \mathrm{H}, \mathrm{H}-2^{\prime}\right), 5.90\left(\mathrm{dd}, J_{\mathrm{vic}}=10.3 \mathrm{~Hz}, J_{\mathrm{gem}}=1.6 \mathrm{~Hz}, 1 \mathrm{H}, \mathrm{H}-3^{\prime}\right.$ trans $), 5.12$ (d, $\left.J_{1-2}=3.4 \mathrm{~Hz}, 1 \mathrm{H}, \mathrm{H}-1\right), 4.66\left(\mathrm{~d}, J_{5-6 \mathrm{exo}}=3.2 \mathrm{~Hz}, 1 \mathrm{H}, \mathrm{H}-5\right), 4.40-4.33(\mathrm{~m}, 2 \mathrm{H}, \mathrm{H}-2$ and H7), $4.18\left(\mathrm{~d}, J_{4-4 \mathrm{a}}=1.1 \mathrm{~Hz}, 1 \mathrm{H}, \mathrm{H}-4 \mathrm{a}\right), 4.12\left(\mathrm{~d}, J_{\mathrm{gem}}=9.9 \mathrm{~Hz}, 1 \mathrm{H}, \mathrm{H}-7\right), 3.78-3.69(\mathrm{~m}, 2 \mathrm{H}$, H-6 endo and H-6 exo), 3.49 (s, $3 \mathrm{H}, \mathrm{H}-8), 2.66$ (dd, $J_{3-4}=10.7 \mathrm{~Hz}, J_{2-3}=6.2 \mathrm{~Hz}, 1 \mathrm{H}, \mathrm{H}-$ 3), $2.19\left(\mathrm{~d}, J_{3-4}=10.7 \mathrm{~Hz}, 1 \mathrm{H}, \mathrm{H}-4\right) ;{ }^{13} \mathrm{C} \mathrm{NMR}\left(\mathrm{CDCl}_{3}\right) \delta 165.2\left(\mathrm{C}, \mathrm{C}-1^{\prime}\right), 146.0(\mathrm{C}$, aromatic), 141.1 (C, aromatic), $141.0\left(\mathrm{C}, 2 \mathrm{C}\right.$, aromatic), $131.4\left(\mathrm{CH}_{2}, \mathrm{C}-3\right), 128.2(\mathrm{CH}$, C-2'), $126.2(\mathrm{CH}$, aromatic), $125.9(\mathrm{CH}$, aromatic), $125.8(\mathrm{CH}$, aromatic), $125.7(\mathrm{CH}$, aromatic), $124.9(\mathrm{CH}$, aromatic), $123.9(\mathrm{CH}$, aromatic), $122.7(\mathrm{CH}$, aromatic), 121.9 (CH, aromatic), $97.0(\mathrm{CH}, \mathrm{C}-1), 76.6(\mathrm{CH}, \mathrm{C}-5), 70.8\left(\mathrm{CH}_{2}, \mathrm{C}-7\right), 70.6(\mathrm{CH}, \mathrm{C}-2), 70.3$ $\left(\mathrm{CH}_{2}, \mathrm{C}-6\right), 59.0\left(\mathrm{CH}_{3}, \mathrm{C}-8\right), 50.5(\mathrm{CH}, \mathrm{C}-4 \mathrm{a}), 49.8(\mathrm{C}, \mathrm{C}-3 \mathrm{a}), 47.5(\mathrm{CH}, \mathrm{C}-4), 36.5$ $(\mathrm{CH}, \mathrm{C}-3)$; HRMS calc. for $\mathrm{C}_{25} \mathrm{H}_{25} \mathrm{O}_{5}[\mathrm{M}+\mathrm{H}]^{+}$405.1696, found 405.1681 .

\section{General procedure for the cycloaddition reaction of 8 and cyclopentadiene}

Acrylic ester 8 (40.4 mg, $0.1 \mathrm{mmol})$ was dried azeotropically with dry benzene and dissolved in the corresponding amount of solvent to give a $0.2 \mathrm{M}$ solution. When the reaction was promoted by Lewis acid the appropriate amount was added under nitrogen and stirred for 20 minutes at the corresponding temperature. Freshly distilled cyclopentadiene ( $82 \mu \mathrm{L}, 1 \mathrm{mmol}$ ) was added dropwise and the mixture was stirred at the temperature and time indicated in Table 2. The cycloaddition reactions carried out without Lewis acid were concentrated after completion to afford a solid residue. The reactions promoted by Lewis acids were quenched by the addition of water $(10 \mathrm{~mL})$ and $\mathrm{HCl} 0.1 \mathrm{~N}(10 \mathrm{~mL})$, then extracted with $\mathrm{CH}_{2} \mathrm{Cl}_{2}(3 \times 30 \mathrm{~mL})$. The combined organic extracts were dried $\left(\mathrm{Na}_{2} \mathrm{SO}_{4}\right)$ and concentrated. The solid residue was purified by flash chromatography to separate the excess of cyclopentadiene and two fractions were obtained: the less polar one contained adducts $9 \mathbf{b}, 9 \mathbf{c}$ and $9 \mathrm{~d}$ and the more polar fraction adduct $9 \mathbf{a}$.

\section{Adduct 9a}

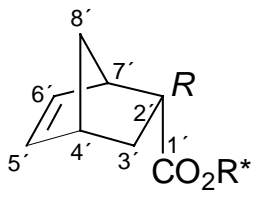

$\mathrm{R}^{\star} \mathrm{OH}=6$

9a 
Colorless oil; $[\alpha]_{\mathrm{D}}=+41.3\left(\mathrm{c}=0.91 ; \mathrm{CHCl}_{3}\right)$; IR (film) $v_{\text {máx }}: 2970,2891,1728(\mathrm{C}=\mathrm{O})$, $1458,1337,1146,1111,1028,748,735 \mathrm{~cm}^{-1} ;{ }^{1} \mathrm{H}$ NMR $\left(\mathrm{CDCl}_{3}\right) \delta 7.46-7.12(\mathrm{~m}, 8 \mathrm{H}$, aromatics), $6.25\left(\mathrm{dd}, J_{5^{\prime}-6}=5.6 \mathrm{~Hz}, J_{4^{\prime}-5}=3.0 \mathrm{~Hz}, 1 \mathrm{H}, \mathrm{H}-5^{\prime}\right), 6.10\left(\mathrm{dd}, J_{5^{\prime}-6}=5.6 \mathrm{~Hz}\right.$, $\left.J_{6^{\prime}-7}=2.6 \mathrm{~Hz}, 1 \mathrm{H}, \mathrm{H}-6^{\prime}\right), 5.01$ (d, $\left.J_{1-2}=3.4 \mathrm{~Hz}, 1 \mathrm{H}, \mathrm{H}-1\right), 4.61$ (d, $J_{5-6 \text { exo }}=3.7 \mathrm{~Hz}, 1 \mathrm{H}, \mathrm{H}-$ 5), 4.25-4.15 (m, $4 \mathrm{H}, \mathrm{H}-2, \mathrm{H}-4 \mathrm{a}$ and H-7), 3.73-3.62 (m, $5 \mathrm{H}, \mathrm{H}-6$ and H-8), 3.28 (broad s, $1 \mathrm{H}, \mathrm{H}-7^{\prime}$ ) , $3.01\left(\mathrm{td}, J_{2^{\prime}-3^{\prime} \mathrm{exo}}=9.4 \mathrm{~Hz}, J_{2^{\prime}-3^{\prime} \mathrm{endo}}=3.9 \mathrm{~Hz}, J_{2^{\prime}-7^{\prime}}=3.9 \mathrm{~Hz}, 1 \mathrm{H}, \mathrm{H}-2^{\prime}\right.$ ), $2.92($ broad s, $1 \mathrm{H}, \mathrm{H}-4$ ) $) 2.38$ (dd, $\left.J_{3-4}=10.5 \mathrm{~Hz}, J_{2-3}=6.0 \mathrm{~Hz}, 1 \mathrm{H}, \mathrm{H}-3\right), 2.13$ (d, $J_{3-}$

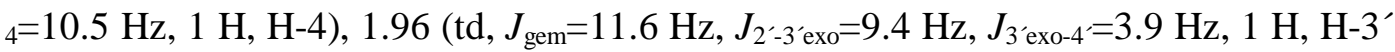
exo), 1.50-1.25 (m, $3 \mathrm{H}, \mathrm{H}-3^{\prime}$ endo and $\left.\mathrm{H}-8^{\prime}\right) ;{ }^{13} \mathrm{C} \mathrm{NMR}\left(\mathrm{CDCl}_{3}\right) \delta 173.8\left(\mathrm{C}, \mathrm{C}-1^{\prime}\right)$, 145.6 (C, aromatic), 140.8 (C, aromatic), 140.7 (C, aromatic), 140.5 (C, aromatic), $137.8(\mathrm{CH}, \mathrm{C}-5)$ ), $132.0(\mathrm{CH}, \mathrm{C}-6)$ ), 126.1 ( $\mathrm{CH}$, aromatic), $125.8(\mathrm{CH}$, aromatic), 125.7 $(\mathrm{CH}, 2 \mathrm{C}$, aromatic), $124.6(\mathrm{CH}$, aromatic $), 123.9(\mathrm{CH}$, aromatic $), 123.4(\mathrm{CH}$, aromatic), $121.8\left(\mathrm{CH}\right.$, aromatic), $96.6(\mathrm{CH}, \mathrm{C}-1), 76.3(\mathrm{CH}, \mathrm{C}-5), 71.3\left(\mathrm{CH}_{2}, \mathrm{C}-7\right), 70.3(\mathrm{CH}, \mathrm{C}-2)$, $70.1\left(\mathrm{CH}_{2}, \mathrm{C}-6\right), 59.0\left(\mathrm{CH}_{3}, \mathrm{C}-8\right), 50.5(\mathrm{CH}, \mathrm{C}-4 \mathrm{a}), 50.3(\mathrm{C}, \mathrm{C}-3 \mathrm{a}), 49.5\left(\mathrm{CH}_{2}, \mathrm{C}-8\right)$, $47.5(\mathrm{CH}, \mathrm{C}-4), 45.5\left(\mathrm{CH}, \mathrm{C}-7^{\prime}\right), 43.3\left(\mathrm{CH}, \mathrm{C}-2^{\prime}\right), 42.4(\mathrm{CH}, \mathrm{C}-4)$ ), $37.4(\mathrm{CH}, \mathrm{C}-3)$, $29.5\left(\mathrm{CH}_{2}, \mathrm{C}-3\right)$.

Adducts endo 9b, exo isomers 9c and 9d

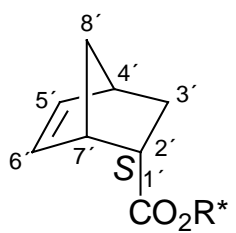

$9 b$

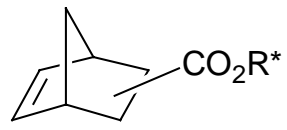

$9 c+9 d$

Colorless oil; IR (film) $v_{\text {máx }}:$ 2970, $1726(\mathrm{C}=\mathrm{O}), 1458,1335,1192,1161,1146,1111$, $748 \mathrm{~cm}^{-1} ;{ }^{1} \mathrm{H}$ NMR $\left(\mathrm{CDCl}_{3}\right) \delta$ 7.44-7.03 (m, aromatics of 9b, 9c and 9d), $6.18\left(\mathrm{dd}, J_{5^{\prime}}\right.$ ${ }_{6}=5.6 \mathrm{~Hz}, J_{4^{\prime}-5}=3.0 \mathrm{~Hz}, \mathrm{H}-5^{\prime}$ of 9b), 6.12 (broad s, H-5' and H-6' of 9c and 9d), 6.00 (dd, $J_{5^{\prime}-6}=5.6 \mathrm{~Hz}, J_{6^{\prime}-7^{\prime}}=2.8 \mathrm{~Hz}, \mathrm{H}-6^{\prime}$ of 9b), 5.05 (d, $J_{1-2}=3.4 \mathrm{~Hz}, \mathrm{H}-1$ of 9c and 9d), $4.96\left(\mathrm{~d}, J_{1-2}=3.4 \mathrm{~Hz}, \mathrm{H}-1\right.$ of 9b), 4.60-4.59 (m, H-5 of 9b, 9c and 9d), 4.34-4.14 (m, H2, H-4a and H-7 of 9b, 9c and 9d), 3.73-3.64 (m, H-6 of 9b, 9c and 9d), 3.56 (s, H-8 of 9b), 3.53 (s, H-8 of 9c and 9d), 3.25 (broad s, H-7' of 9b), 3.08-2.91 (m, H-4' and H-7' of 9c and 9d, H-4' of 9b and H-2' of 9b), 2.57-2.43 (m, H-3 of 9b, 9c and 9d), 2.352.28 (m, H-2 of 9c and 9d), 2.16-1.87 (m, H-4 and H-3' exo of 9b, 9c and 9d), 1.571.25 (m, H-3' endo and $\mathrm{H}-8^{\prime}$ of 9b, 9c and 9d); ${ }^{13} \mathrm{C} \mathrm{NMR}\left(\mathrm{CDCl}_{3}\right) \delta 175.3$ and 175.1 (2C, C-1' of 9c and 9d), 173.8 (C, C-1' of 9b), 145.8 (C, aromatic, 9b, 9c and 9d), 141.0 (C, aromatic, 9b, 9c and 9d), 140.9 (C, aromatic, 9b, 9c and 9d), 140.8 (C, aromatic, 9b, 9c and 9d), 138.2 and $137.9\left(2 \mathrm{CH}, \mathrm{C}^{-5^{\prime}}\right.$ of $9 \mathrm{c}$ and $\left.9 d\right), 137.4\left(\mathrm{CH}, \mathrm{C}-5^{\prime}\right.$ of 9b), 135.7 and 135.4 (2 CH, C-6' of 9c and 9d), $132.6\left(\mathrm{CH}, \mathrm{C}^{-6} 6^{\prime}\right.$ of 9b), $126.1(\mathrm{CH}$, aromatic, 9b, 9c and 9d), $125.8(\mathrm{CH}$, aromatic, 9b, 9c and 9d), $125.7(\mathrm{CH}, 2 \mathrm{C}$, aromatic, 9b, 9c and 9d), $124.8(\mathrm{CH}$, aromatic, 9b, 9c and 9d), $123.9(\mathrm{CH}$, aromatic, 9b, 9c and 9d), $122.9(\mathrm{CH}$, aromatic, 9b, 9c and 9d), $121.8(\mathrm{CH}$, aromatic, 9b, 9c and 9d), 
96.8 (CH, C-1 of 9b, 9c and 9d), $76.5\left(\mathrm{CH}, \mathrm{C}-5\right.$ of 9b, 9c and 9d), $71.0\left(\mathrm{CH}_{2}, \mathrm{C}-7\right.$ of 9b, 9c and 9d), $70.2\left(\mathrm{CH}_{2}, \mathrm{C}-6\right.$ of 9b, 9c and 9d), $70.0(\mathrm{CH}, \mathrm{C}-2$ of 9b, 9c and 9d), 59.0 $\left(\mathrm{CH}_{3}, \mathrm{C}-8\right.$ of 9b, 9c and 9d), $50.5(\mathrm{CH}, \mathrm{C}-4 \mathrm{a}$ of 9b, 9c and 9d), $50.0(\mathrm{C}, \mathrm{C}-3 \mathrm{a}$ of 9b, 9c and 9d), $49.4\left(\mathrm{CH}_{2}, \mathrm{C}-8^{\prime}\right.$ of 9b), $47.5(\mathrm{CH}, \mathrm{C}-4$ of 9b, 9c and 9d), 46.9 and $46.4(2 \mathrm{CH}$, C-7' of 9c and 9d), 46.3 and $46.0\left(2 \mathrm{CH}_{2}, \mathrm{C}^{\prime} 8^{\prime}\right.$ of $9 \mathrm{c}$ and $\left.9 \mathrm{~d}\right), 45.7\left(\mathrm{CH}, \mathrm{C}-7^{\prime}\right.$ of $\left.9 \mathrm{~b}\right)$, $43.3\left(\mathrm{CH}, \mathrm{C}-2^{\prime}\right.$ of $\left.9 \mathbf{b}\right), 43.1\left(\mathrm{CH}, \mathrm{C}^{-2} 2^{\prime}\right.$ of $9 \mathbf{c}$ and $\left.9 \mathrm{~d}\right), 42.5\left(\mathrm{CH}, \mathrm{C}^{\prime} 4^{\prime}\right.$ of $\left.9 \mathbf{b}\right), 41.6(\mathrm{CH}$, C-4' of 9c and 9d), $36.9(\mathrm{CH}, \mathrm{C}-3$ of $9 \mathbf{b}, 9 \mathbf{9}$ and $9 d), 30.1\left(2 \mathrm{CH}_{2}, \mathrm{C}^{\prime} 3^{\prime}\right.$ of $9 \mathrm{c}$ and $\left.9 d\right)$, $29.1\left(\mathrm{CH}_{2}, \mathrm{C}^{\prime} 3^{\prime}\right.$ of $\left.\mathbf{9 b}\right)$.

\section{Hydrolysis of cicloadduct 9a}

Adduct 9a (95 mg, $0.202 \mathrm{mmol}$ ) was dissolved in THF- $\mathrm{H}_{2} \mathrm{O} 2: 1(3 \mathrm{~mL})$ and $\mathrm{LiOH}_{2} \mathrm{H}_{2} \mathrm{O}$ $(51,0 \mathrm{mg}, 1.215 \mathrm{mmol})$ was added. The reaction was stirred at room temperature for 7 days at $60^{\circ} \mathrm{C}$. The solution was neutralized with $\mathrm{HCl} 0.1 \mathrm{~N}$ to reach $\mathrm{pH}=4$ and extracted with ether $(5 \times 40 \mathrm{~mL})$. The combined organic extracts were dried $\left(\mathrm{Na}_{2} \mathrm{SO}_{4}\right)$ and the solvent was evaporated. The resulting residue was purified by flash chromatography to obtain the chiral auxiliary $6(69.4 \mathrm{mg}, 0.20 \mathrm{mmol}, 98 \%)$ and 2-norbornene-5-carboxylic acid (12) $(21.8 \mathrm{mg}, 0.16 \mathrm{mmol}, 79 \%)$. The optical rotation of 12 was $[\alpha]_{\mathrm{D}}+149.9(c$ $\left.0.38, \mathrm{Cl}_{3} \mathrm{CH}\right)\left[\right.$ lit. $^{3}-151.6\left(c 2.0, \mathrm{Cl}_{3} \mathrm{CH}\right)$ ], indicating that cycloadduct 9a was the $(2-R)$ enantiomer.

\section{NMR spectra of 8 with $\mathbf{E t}_{2} \mathbf{A l C l}$}

Complexation of $\mathbf{8}$ with Lewis acids: for the ${ }^{1} \mathrm{H}$ NMR experiments the acrylic ester $\mathbf{8}$ (30 $\mathrm{mg}, 0.074 \mathrm{mmol}$ ) was dissolved in dry $\mathrm{CDCl}_{3}(0.4 \mathrm{~mL})$ in a NMR tube under nitrogen. The appropriate amount of $\mathrm{Et}_{2} \mathrm{AlCl}(1.8 \mathrm{M}$ in toluene) was added to the solution. Compound $\mathbf{8}$ showed to be stable during the time required for the acquisition of the spectra.

\footnotetext{
${ }^{3}$ Chang, H.; Zhou, L.; McCargar, R. D. Mahmud, T.; Hirst, I. Org. Process Research \& Development, 1999, 3, 289.
} 


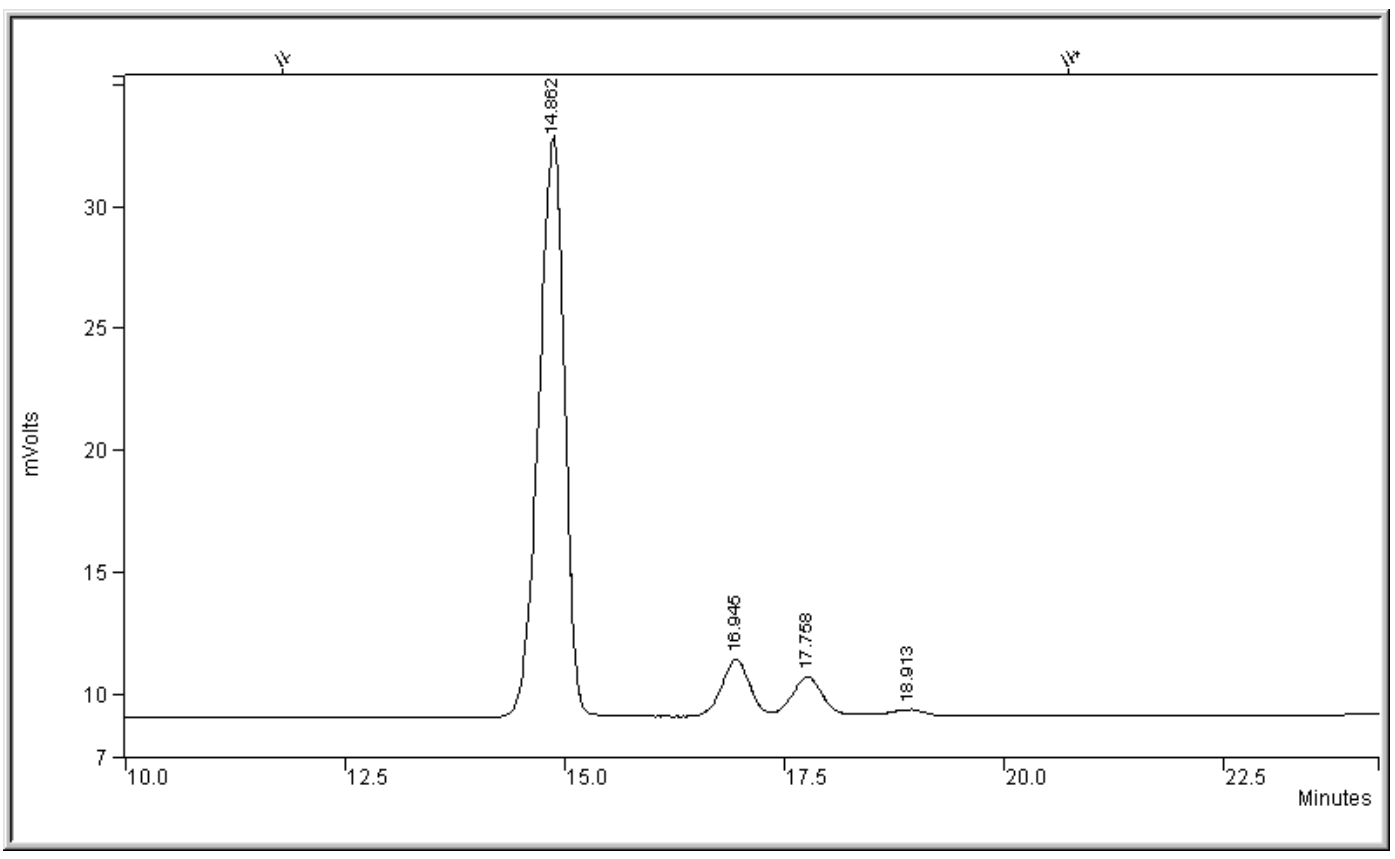

HPLC Chromatogram of the mixture of adducts.

\begin{tabular}{|c|c|}
\hline Retention Times (min) & Adduct \\
\hline 14.86 & 9a \\
\hline 16.94 & 9b \\
\hline 17.75 and 18.91 & 9c and 9d \\
\hline
\end{tabular}


${ }^{1} \mathrm{H}$ and ${ }^{13} \mathrm{C}$ NMR Spectra of compound 4
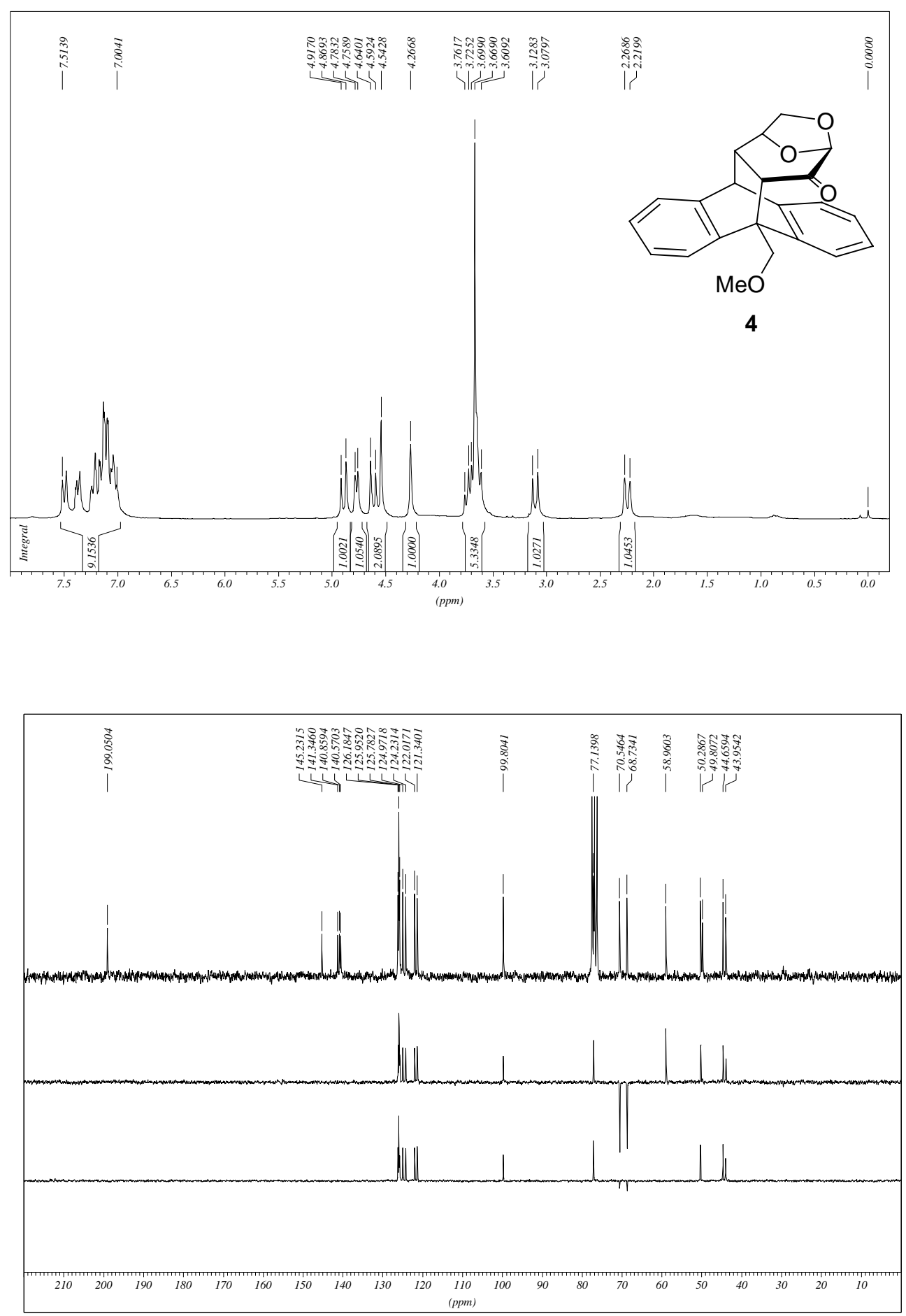
${ }^{1} \mathrm{H}$ and ${ }^{13} \mathrm{C}$ NMR Spectra of compound 5
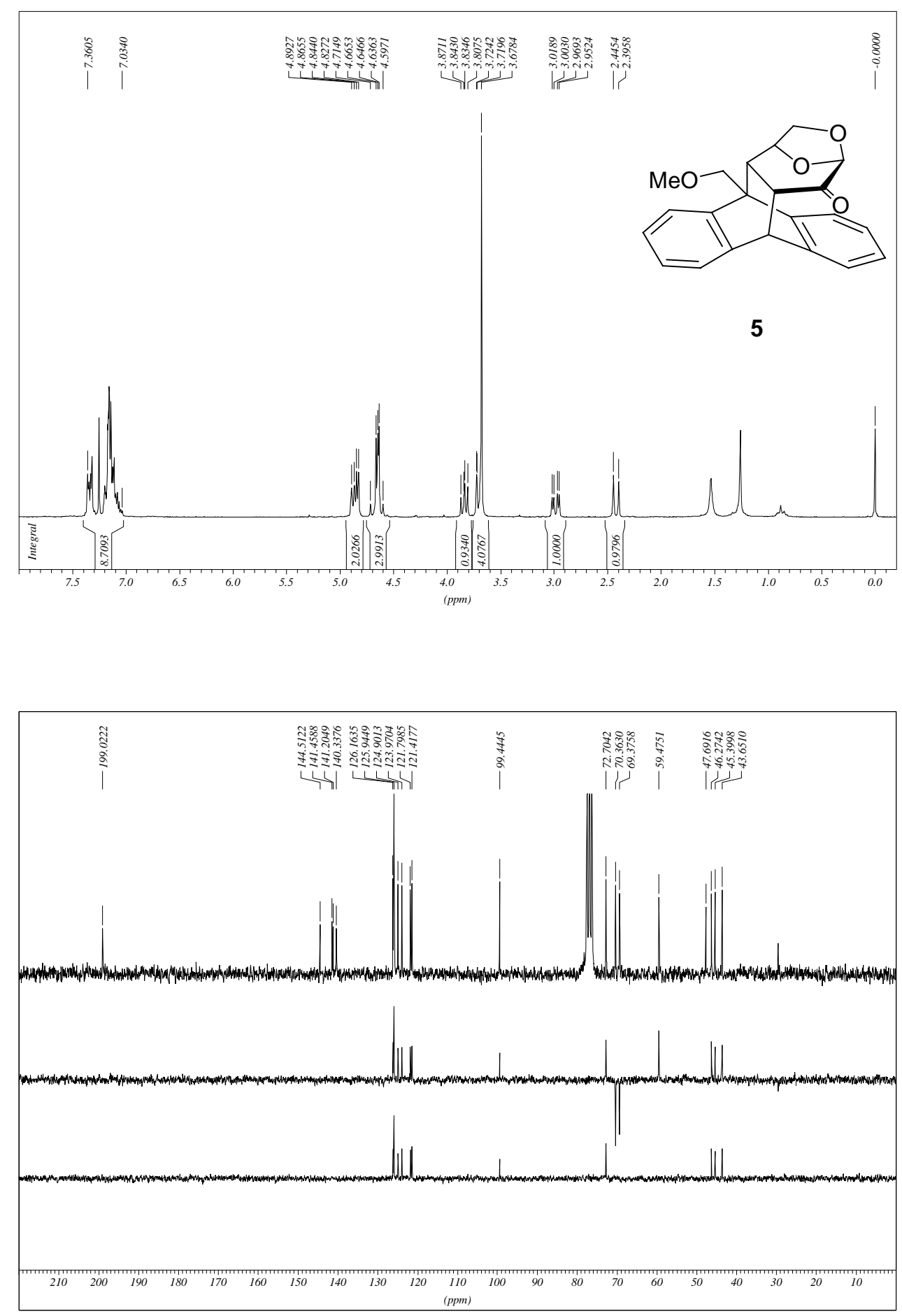
${ }^{1} \mathrm{H}$ and ${ }^{13} \mathrm{C}$ NMR Spectra of compound 6
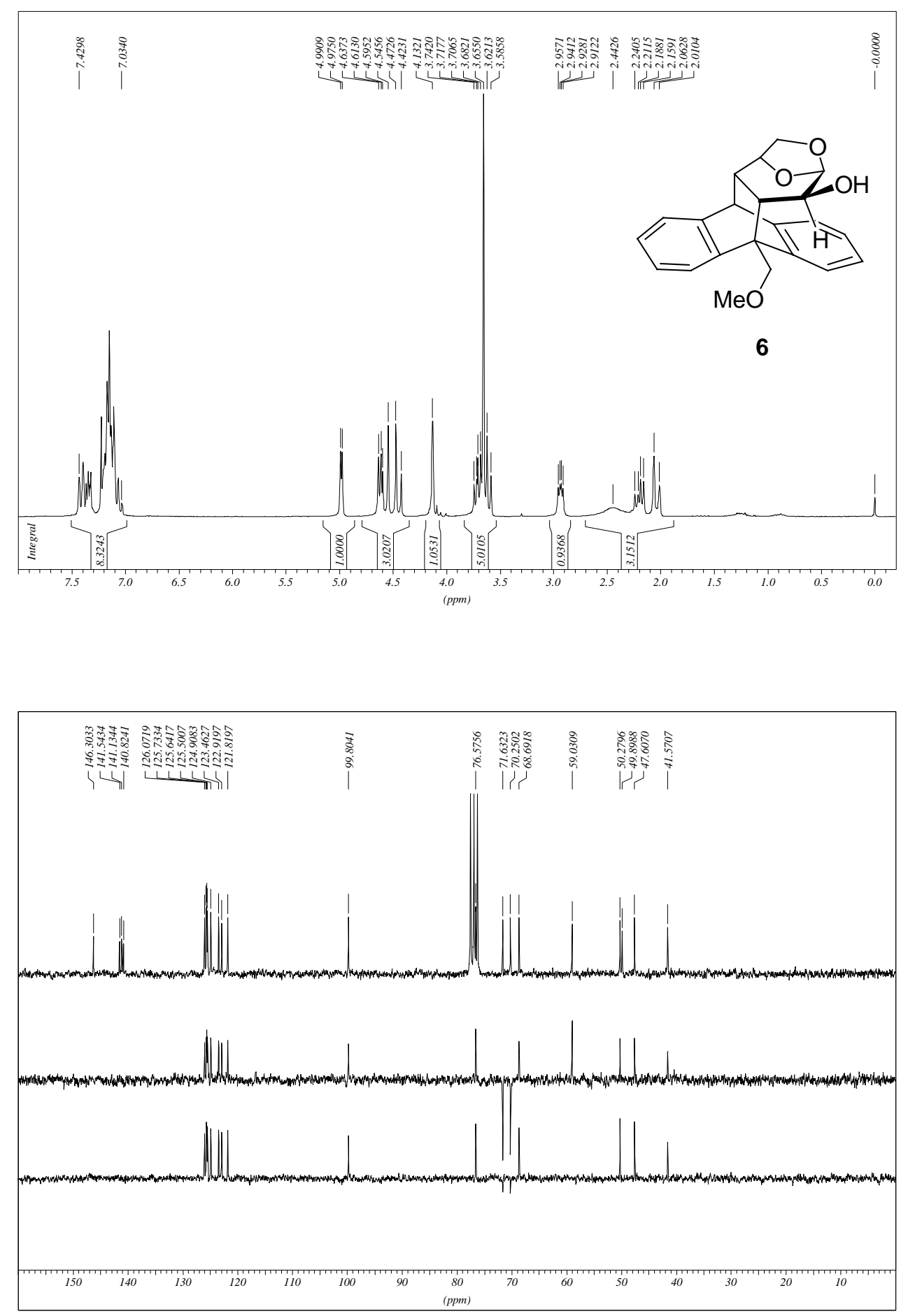
${ }^{1} \mathrm{H}$ and ${ }^{13} \mathrm{C}$ NMR Spectra of compound 7
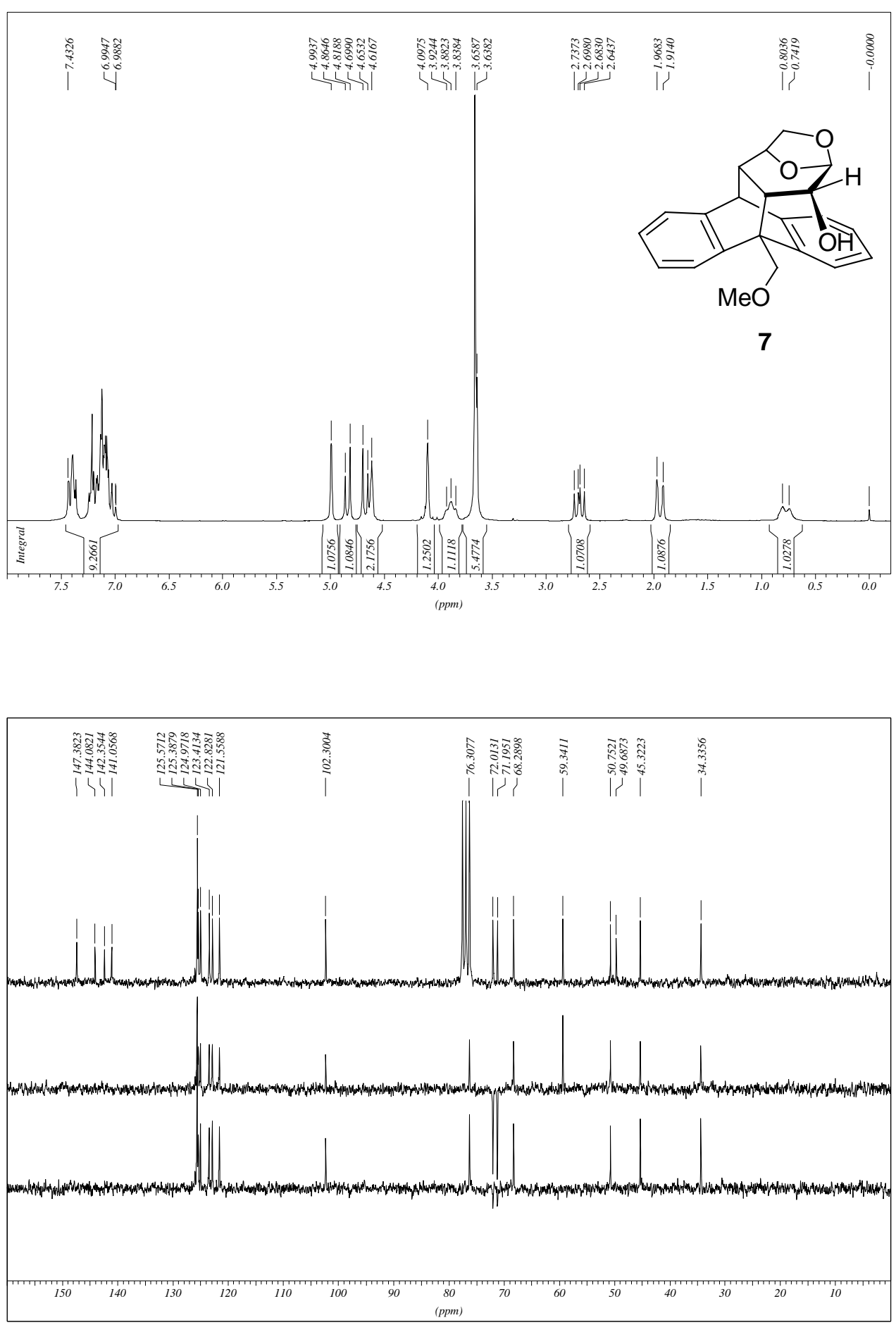
${ }^{1} \mathrm{H}$ and ${ }^{13} \mathrm{C}$ NMR Spectra of compound 8
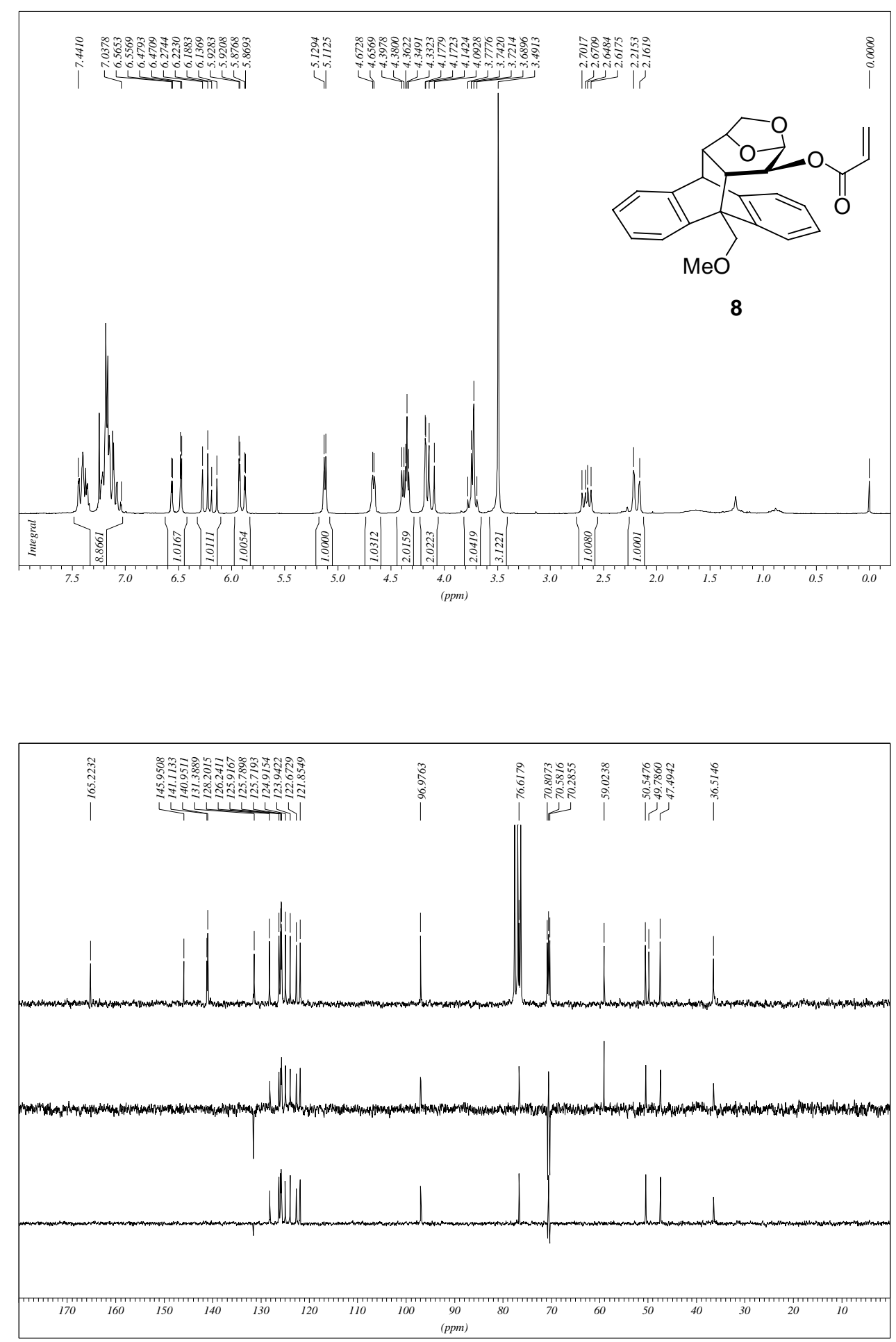
${ }^{1} \mathrm{H}$ and ${ }^{13} \mathrm{C}$ NMR Spectra of compound $9 \mathbf{a}$
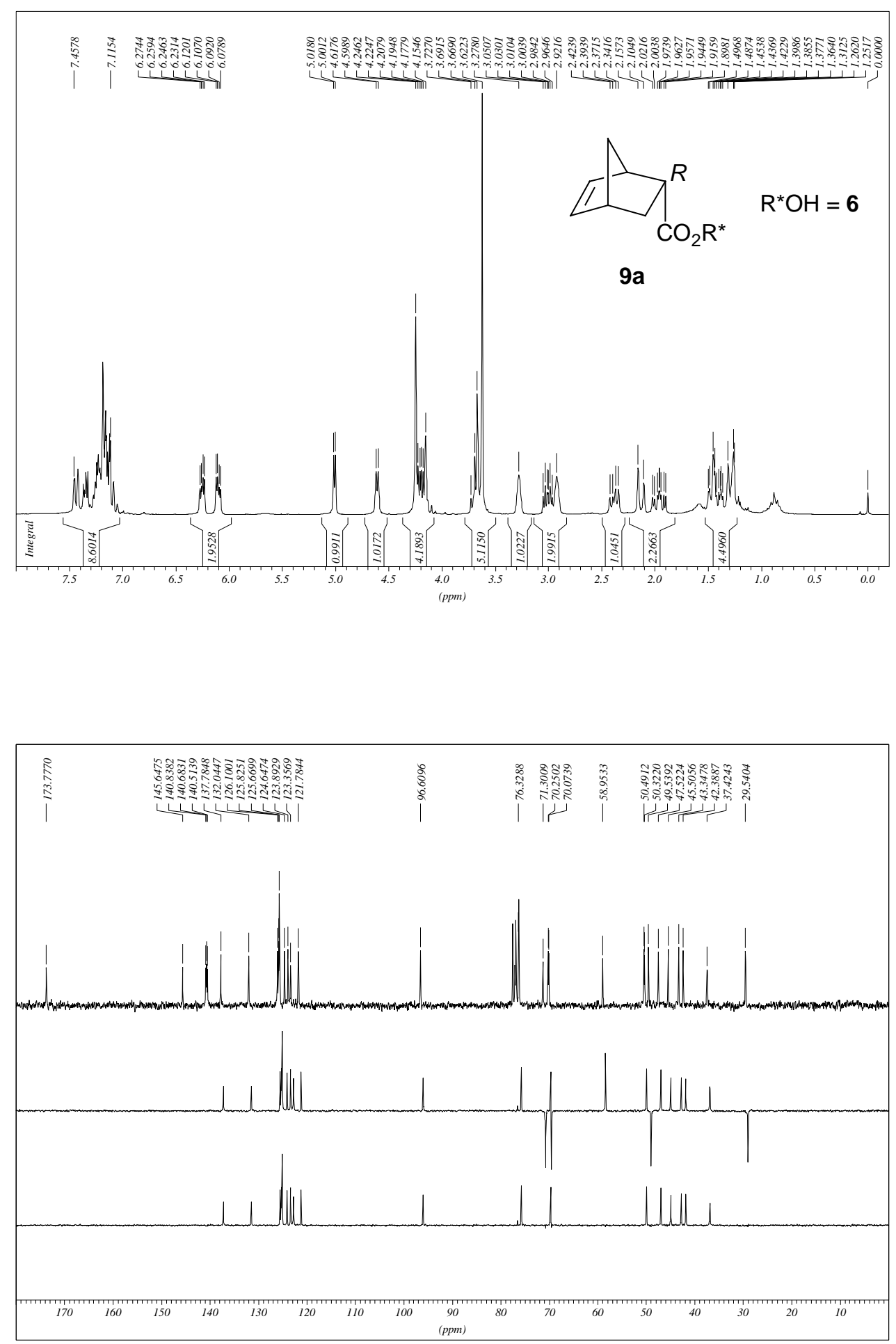


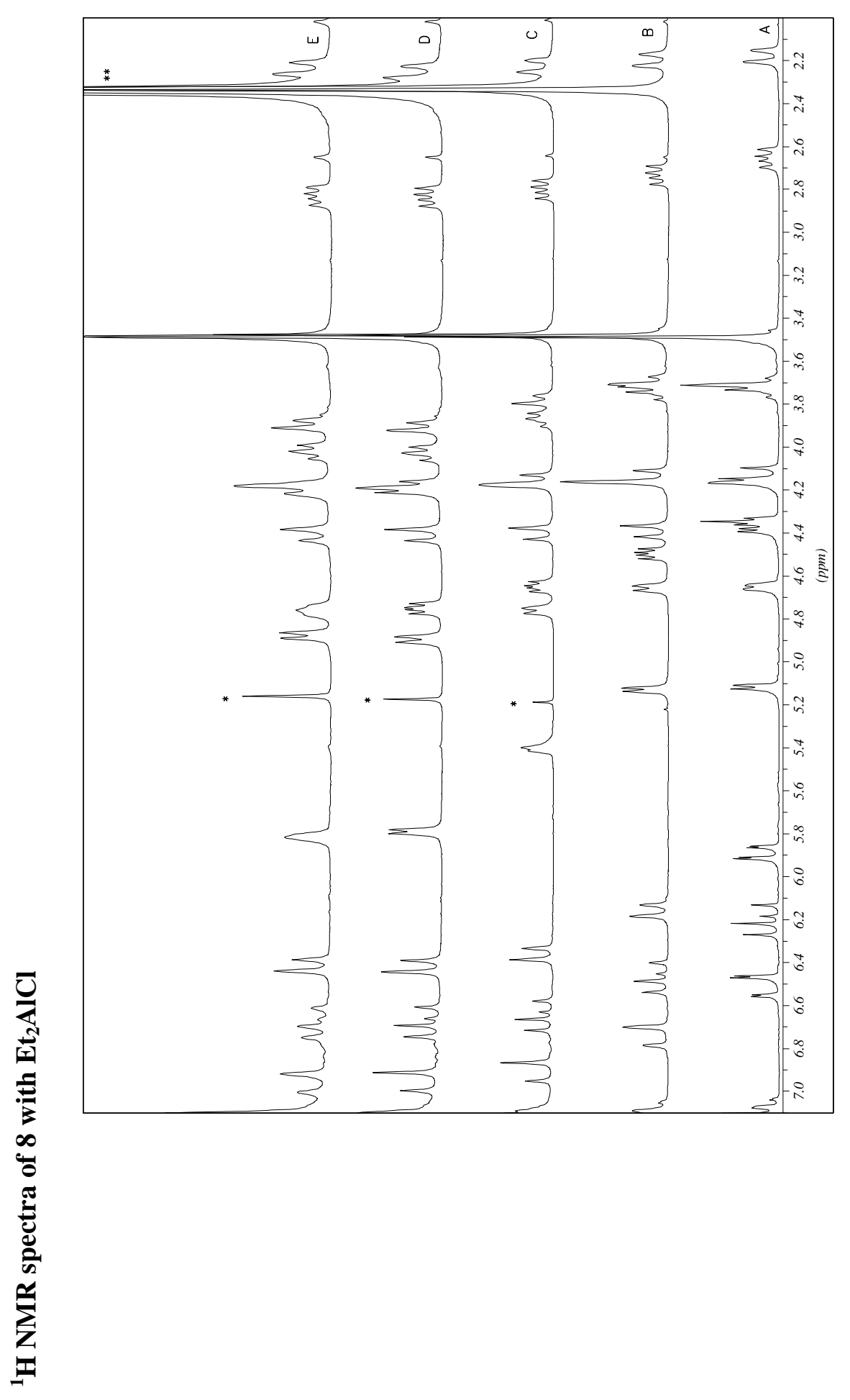

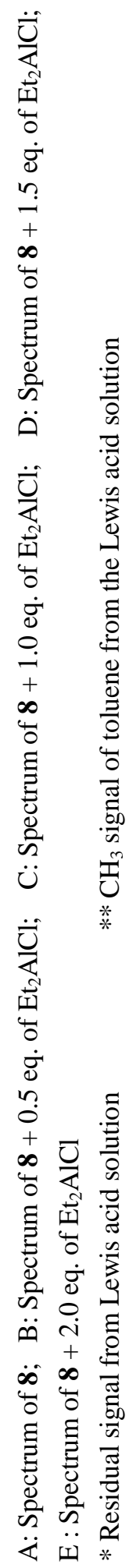

